\title{
LA EPISTEMOLOGÍA DEL MAESTRO: UN PODEROSO REFERENTE EN LA ENSEÑANZA DE LA BIOLOGÍA
}

\author{
Lilia Reyes-Herrera
}

\begin{abstract}
The main purpose of this work was to explore a teacher referents for teaching and learning biology while developing an ecology unit for six graders in a public school in Bogotá. Why does the teacher teaches in that way? What are his or her referents for teaching? Following the ethnographic methodology proposed by Guba and Lincoln (1989) we identify that:

* In this class, there were deep power imbalances in terms of control and authority that makes the teacher the main source of knowledge. The central vision was the authority of teacher's explanations and the control of the activities.

* In this context, biology was assimilated in general, as the search for truth. A need to probe what the teacher said was evident.

* There was a pervasive idea about the use of scientific method in biology with frequent explanations by the teacher about the steps to follow

These and other similar conceptions of this teacher were coherent with a positivist philosophy and constituted a powerful referent for teacher and students work. If we want an alternative in biology teaching, teachers must implement their own project to reflect on their own teaching and learning. As long as the teacher make explicit his or her own principles he would be able to reflect and consider possibilities to change. In this way critical inquiry and reflection about his or her own actions promise the construction of new theoretical referents and new actions in the teaching and learning of biology.
\end{abstract}

\section{PALABRAS CLAVES}

Epistemología, desarrollo social, autonomía, etnografía, creencias, conocimientos.

\section{INTRODUCCIÓN}

Mejorar la enseñanza de la ciencia y la tecnología es uno de los grandes desafíos para toda la humanidad, no sólo en el momento actual sino también más allá del año 2000. Particularmente para nosotros, la comunidad comprometida con la educación en biología, este gran reto cobra vital importancia. Recientemente los desarrollos de la biología y la tecnología han alcanzado grandes dimensiones que para su adecuada comprensión imponen un requisito de permanente actualización teórica y práctica a todos los ciudadanos. De una u otra manera, todos los seres humanos somos afectados por los adelantos y limitaciones de la ciencia (biología) y la tecnología, por lo tanto, para poder tomar decisiones mejor informadas en el campo de la biología, su enseñanza, y su

\footnotetext{
"Profesora Universidad Pedagógica Nacional, Colombia.
} 
aprendizaje, es muy importante que todos los individuos tengan acceso a educación formal o no formal en biología, conscientes de que existen otros enfoques alternativos al pensamiento que domina actualmente en la mayoría de nuestras aulas de clase.

Por otra parte existe un marcado contraste entre el aprendizaje de los estudiantes en biología y las creencias de los educadores ya que existe una fuerte coherencia entre la forma en que el maestro enseña y sus concepciones acerca de la biología, la enseñanza y el aprendizaje. Esta situación unida a la dificultad de cambio presentada por algunos docentes es persistente en todos los niveles de la educación, desde el preescolar hasta la educación superior (Tobin, 1993, Reyes-Herrera, 1996). Entender el pensamiento del maestro de biología acerca del aprendizaje y la enseñanza es uno de los factores críticos para proveer y promover mejores ambientes de aprendizaje de la biología. Esta situación es válida no solo para los docentes de biología de todos los niveles, sino también para todos los investigadores educativos y demás personas responsables de la actualización, la formación de maestros y la generación de políticas educativas en este campo.

Si los maestros que se identifiquen con David (el maestro de este estudio) reconocen sus propias creencias y desarrollan proyectos y experiencias educativas alternativas, habrá mejor posibilidad de que se produzca un mayor desarrollo profesional y un apreciable enriquecimiento intelectual mutuo de maestros, estudiantes e instituciones educativas y se promuevan nuevas ideas, acciones y modelos hacia el mejoramiento de la enseñanza de la Biología.

\section{ANTECEDENTES}

La educación colombiana a través del tiempo se ha caracterizado generalmente por aplicar casi que directamente, modelos diseñados y desarrollados en otros países, los cuales por razones obvias no responden ni a las necesidades, ni a las condiciones, ni a la cultura propia de las diferentes regiones de nuestro país, esta situación ha traído como consecuencia que se repitan los paradigmas educativos más corrientes en otros países y latitudes. Como resultado de lo anterior, diferentes investigadores han identificado la persistencia de un modelo transmisionista de la educación, caracterizado por una excesiva atención a los contenidos y la reproducción memorística de la información. Adicionalmente en muchos casos los profesores tienen una concepción de ciencia como algo ya acabado, y aunque tratan de aplicar soluciones a los problemas detectados en la mayoría de los casos estas respuestas, debido a los fundamentos en que se apoyan, no contribuyen a desarrollar autonomía ni creatividad bien sea en el maestro o en sus estudiantes. Los factores mencionados limitan las posibilidades de desarrollo y competencia de nuestro país dado que un tipo de educación ajeno a las necesidades y al contexto propio no favorece el desarrollo integral de la persona ni de la sociedad a la cual pertenece.

A nivel internacional, tal como está planteado en el documento Ciencia para todos los Americanos (Rutherford \& Ahlgren, 1990, p.v. Science for all Americans) la educación en ciencia -lo que significa educación en ciencia, matemática y tecnología- debe ayudar a los estudiantes a desarrollar el entendimiento y los hábitos mentales necesarios para ser seres humanos compasivos, capaces de pensar por si mismos y enfrentar la vida". Se hace entonces necesario que la educación en ciencias (para nuestro caso en biología) contribuya no solamente al desarrollo individual sino también al colectivo proveyendo elementos que faciliten al ser humano interacciones con sus semejantes y con su entorno, 
para mantener la voluntad política de sus comunidades favoreciendo situaciones de equidad, justicia y bienestar para nuestros pueblos.

De la misma manera, en Colombia se propende por una nueva concepción de la ciencia (biología), de su enseñanza y de su aprendizaje a este respecto el CONPES (1994 p. 1) enfatiza que "La ciencia \{biología\} entendida como una forma de conocimiento orientada a comprender, explicar y transformar tanto al hombre como al entorno en que vivimos, ha dejado de ser una actividad de interés exclusivo de los científicos, para convertirse en componente inseparable de la cultura universal y del desarrollo económico contemporáneo. En este nuevo contexto, la ciencia y la tecnología se han convertido en un factor de cambio y de crecimiento económico. Debidamente orientadas se traducen en desarrollo y bienestar para los pueblos". Por lo tanto el desarrollo y bienestar de Colombia compromete nuestra participación pensante, en unión con nuestros semejantes, a fin de proteger el futuro de nuestra nación pues ese futuro está delimitado en gran parte por el carácter y la calidad de la educación que estemos desarrollando en todas nuestras instituciones escolares. A partir de estos planteamientos surge como propósito fundamental la investigación en educación en biología, la cual está asociada con el reto al pensamiento del profesor y a los planteamientos dominantes acerca de la relación de cada ciudadano con la ciencia, la matemática y la tecnología. Esto implica por una parte auscultar creencias propias de maestros y estudiantes en torno a la ciencia-biología, su naturaleza y sus relaciones con la sociedad y por otra explorar nuevas alternativas que contribuyan a satisfacer las necesidades de aprendizaje de la biología en todos los ciudadanos.

\section{JUSTIFICACIÓN}

Una posible educación de calidad en nuestro medio implicaría el desarrollo de modelos propios que respondan a nuestra cultura, es decir a la idiosincrasia de nuestras comunidades y que favorezcan la autonomía y el auto desarrollo de los estudiantes y demás agentes educativos Por ello, uno de los retos que afronta la educación actual en Colombia, es la formación de recursos humanos con capacidad de producción de conocimientos científicos y tecnológicos para enfrentar sus problemas y producir nuevos bienes y servicios, a fin de asegurar el bienestar de su población y participar en la dinámica mundial como nación independiente y competitiva. Por tanto se requiere la apropiación social de la ciencia y la tecnología y la formación de ciudadanos capaces de comprenderlas y producir conocimientos en estas áreas tal como lo reclama la Misión de Ciencia, Educación y Desarrollo (CONPES.1994).

Investigaciones recientes en educación han explorado la relación entre las creencias del maestro y la enseñanza de las Ciencias naturales (Cronin-Jones, 1991, Caicedo, 1992; Tobin, 1993; Tobin \& Tippins, 1993; Salcedo, 1994; Tobin, 1995; Reyes-Herrera, 1996). Últimamente McRobbie and Tobin (1995), notaron en su estudio que a pesar de presentarse una fuerte influencia entre las creencias del maestro y de los estudiantes sobre las prácticas del aula también había una infraestructura que dependía de elementos de la cultura predominante. Esta infraestructura no es otra cosa que el contexto cultural determinado por las vivencias cotidianas del maestro que ayudan a delinear y darle forma a ese "espacio de encuentro" entre el estudiante y el conocimiento científico en búsqueda de explicaciones y significados. En cierta manera el contexto cultural moldea el quehacer docente y define las oportunidades de aprendizajes significativos. 
Pero el contexto de enseñanza esta integrado a su vez por varios elementos que se entrelazan formando una red de conexión entre ellos. Estos elementos son las creencias del maestro; las metas e intereses del maestro con respecto a sus estudiantes, con respecto a si mismo y con respecto al desarrollo del propio currículum, y el comportamiento o roles del maestro al desarrollar el currículum. Todos estos elementos coordinados de manera significativa y coherente permiten al maestro tomar conciencia del desarrollo de sus acciones. Acción en este estudio no es sinónimo de comportamiento. La acción consciente del maestro coordina armónicamente los elementos anteriores en un contexto específico.

\section{PROPÓSITO}

El propósito central de este estudio fue, explorar el acercamiento de un maestro y sus estudiantes al conocimiento científico mientras el docente desarrollaba una unidad de ecología para sexto grado en una institución pública en Bogotá. ¿Por qué, el maestro enseña de la forma que lo hace? ¿En qué medida contribuye la educación en biología a preparar a los hombres y mujeres de hoy para vivir una vida plena y responsable? ¿Cuáles son los referentes que guían las acciones de maestros y estudiantes al construir ambientes en los cuales se pueda aprender biología? ¿Qué concepciones tiene el maestro acerca de la biología, la enseñanza, el aprendizaje? Respuestas a estas y otras preguntas semejantes constituyen los propósitos de esta investigación.

\section{METODOLOGIA}

La estrategia de investigación seguida en este estudio está relacionada con enfoques llamados: etnográfico, cualitativo, de observación participativa, de estudio de caso, fenómenológico, constructivista o interpretativo, que son esenciales en el proceso de interpretar las acciones de este maestro Todos estos enfoques están estrechamente relacionados y son relativamente nuevos en el campo de la investigación en la enseñanza de las disciplinas especificas. Su interés central que puede agruparse bajo el apelativo de interpretativo, es la búsqueda de significados en la vida social del aula y de la escuela y su elucidación y exposición por parte del grupo de investigación del cual hacen parte central los maestros e investigadores.

Como lo afirma Erickson (1996), su principal criterio de validez es el significado inmediato y local de las acciones de los participantes ( $p$ 119). Los datos de este estudio se seleccionaron de transcripciones de videos de clase, entrevistas y notas de campo. Al documentar detalles concretos de la práctica se puede entender de mejor manera qué está sucediendo en el aula, específicamente desde el punto de vista del maestro También se puede entender el significado local que lo que está sucediendo tiene para las personas involucradas. Por lo tanto, coherentemente con el problema a investigar se empleó la metodología propuesta por Guba y Lincoln (1989) que permite la interpretación de la dinámica realidad que representa el aprender y enseñar biología cada día

\section{RELACIONES ENTRE CONOCIMIENTO Y ENSEÑANZA DE LA BIOLOGÍA}

Las concepciones de los maestros de biología sobre conocimiento, biología, verdad y método son muy variadas y además están poco documentadas en Colombia En este 
estudio se identifican algunos patrones de relaciones entre conocimiento y poder, conocimiento y verdad y conocimiento y método.

\section{Conocimiento, control y autoridad}

Relaciones de poder del maestro fueron identificadas en términos de control y autoridad durante el desarrollo de esta asignatura Estas relaciones son bien marcadas y hacen que el maestro sea considerado como fuente principal de conocimiento. La visión dominante fue la explicación con autoridad y el control de las actividades a realizar que se puede evidenciar en el siguiente segmento de una de las entrevistas.

David: Yo decido las actividades pero ya en situación de clase fácilmente las cambio o doy libertad al estudiante para decidirlas según el interés o la necesidad del momento. El dar la respuesta correcta impide el desarrollo mental del alumno, el guiarlos u orientarlos enriquece procesos mentales de análisis, aplicación, relación, etc. Es casi imposible darles más libertad a los estudiantes porque no están acostumbrados a manejar su libertad, lo que hace que muchas veces lo conviertan en libertinaje. Por otra parte las condiciones del colegio y de muchos compañeros de trabajo, así como la rigidez de los supuestos acuerdos en torno al programa no facilita. Yo controlo el ritmo de la clase para proponer más o menos tiempo según el interés que muestren los alumnos. Son los estudiantes los que al analizar.., se encargan de la clase, yo lo hago indirectamente al orientar sus procesos.

Bien vale la pena preguntarnos qué formas deben tomar las relaciones entre profesores y estudiantes, sí lo que queremos es el desarrollo de ambientes de aprendizaje más apropiados, es decir donde el estudiante tenga mayor libertad de aprender con autonomía acerca de situaciones relevantes. ¿Qué clase de relaciones serán más apropiadas para promover el mejoramiento de la educación en biología si queremos promover en nuestros estudiantes mejor capacidad para resolver problemas y que el estudiante tenga la posibilidad real de superar a su maestro?

\section{Conocer: un desafío a la verdad absoluta}

En esta clase existe una gran tendencia a considerar la biología como la búsqueda de la verdad y la comprobación de esa verdad relegando en gran medida la búsqueda de significados. Hablando acerca de las funciones del ecosistema el diálogo se desarrolla de la siguiente forma:

David continúa: Entonces vamos a tratar de hacer una lista de qué se les ocurrió a ustedes pensar que podrían ser las funciones del ecosistema. 'Adelante se oye equilibrio'. David continúa: entonces me dicen que mantener el equilibrio. [Y escribe equilibrio en el tablero] ¿Cuál otra? Fernando responde: hace posible la vida. David: entonces facilita la vida de los organismos, ¿cual otra? Carlos Andrés ofrece: desarrollo de los seres vivos, David: entonces facilita el desarrollo de los seres vivos. Patricia: hábitat, David complementa: constituir un hábitat para los organismos, y tú ¿qué nos dices que no te he oído la voz? Guillermo contesta: esparcimiento para los seres vivos. [David ha copiado en el tablero las palabras claves que han dicho los estudiantes, hasta ahora hay una lista con las siguientes palabras: equilibrio, facilita la vida, desarrollo, hábitat, esparcimiento]. ¿Qué otra se les ocurrió? Relacionarse, 
contesta Ana María. David: entonces permite las relaciones entre los diferentes seres, ¿Qué otras funciones del ecosistema que no están allá? ¿A ver? Otro estudiante contesta: alimento y David complementa: dar alimento a los seres vivos. David pregunta: ¿Quedó claro? Y algunos estudiantes responden sí. Se nota un ambiente de tranquilidad y aceptación en clase y David pasa a otra actividad.

En esta clase es común que los estudiantes contesten con una o pocas palabras que son consideradas como verdades absolutas, y es David quien complementa afirmando o desconfirmando lo dicho por el estudiante, se nota que la verdad la posee el libro de donde memorizaron las palabras claves o en el mejor de los casos David es quien posee esa verdad. No hay lugar a cuestionamientos ni a interpretación, ni a ampliación de lo que se está diciendo por parte de los estudiantes. La participación de los estudiantes es mínima y se confía en el conocimiento del maestro como suprema verdad. Una crítica a los fundamentos epistemológicos de la biología parece presentar un reto a la concepción de verdades únicas y absolutas y tal como lo argumentan Aronowitz and Giroux (1993), parece posible que el conocimiento científico puede ser disponible y accesible para todos los estudiantes silo bajamos de su pedestal absolutista. (pág. 22).

\section{Método científico y conocimiento}

Prevalece la idea de el "método científico" para el trabajo en biología siendo frecuentemente el maestro quien indica claramente las etapas a seguir para el desarrollo de experiencias. David cree que si los estudiantes siguen la metodología científica ellos aprenderán biología en una forma autónoma. David considera que el método científico involucra las siguientes etapas: observación, clasificación, identificación del problema, formulación de hipótesis y finalmente verificación de las hipótesis para terminar en la formulación de nuevas preguntas. El siguiente segmento de una clase nos ayuda a entender como David entiende el proceso de investigación.

En esta clase David estaba preocupado por que sus estudiantes entendieran el proceso de investigación científica. Es interesante observar que la pregunta problema fue, asignada por David como tarea en la clase anterior de tal manera que se esperaba que los estudiantes buscaran respuestas apropiadas en sus libros para resolver su tarea. La pregunta problema asignada por David fue ¿Cuáles son las funciones del ecosistema? David procede de la siguiente forma.

Ahora al primer punto de la tarea, A ver: Funciones, ¿Qué se les ocurrió a ustedes pensar que eran las funciones del ecosistema? Primero quiero que caigan en cuenta de una cosa, cuando hicimos ese cuadro [refiriéndose a la lista de seres vivos y no vivos] con qué viene a ser igual en el proceso metodológico? Sara, una de las estudiantes responde: con la observación, David continúa: Cuando yo les dejé la tarea. ¿qué viene a ser esa tarea?

Varios estudiantes responden: problemas. David confirma: una pregunta problema. Ahora qué, hicieron ustedes cuando trataron de resolverlas? Varios estudiantes contestan: hipótesis. David continúa: formulación de hipótesis.

Las hipótesis fueron deducidas por David a partir de palabras claves de los estudiantes en sus intentos de contestar las preguntas, ejemplo: cuando el estudiante dice "equilibrio" David reconstruye mantener el "equilibrio". Así que la prueba teórica de la hipótesis también fue, dominada por David. Este enfoque de enseñanza centrado típicamente en el maestro hace que los estudiantes contesten como en un juego de adivinanzas 
contestando la mayoría de las veces con una palabra o con una frase muy corta. Es importante anotar que los estudiantes no hicieron ninguna pregunta ni ofrecieron comentarios adicionales en toda esta sección, por lo tanto visiones críticas fueron muy raras en esta clase.

También es importante distinguir entre las teorías enunciadas por el maestro y las teorías utilizadas al momento de realizar sus acciones pues observaciones cuidadosas de la labor del maestro han revelado discrepancias entre la teoría del maestro (lo que se dice) y su práctica educativa (lo que se hace). Como lo discute Young (1992, p. 18), maestros que aseguraron tener una visión crítica de la enseñanza se encontraron ellos mismos enseñando autoritariamente algunas veces o proveyendo fórmulas metodológicas paso por paso a estudiantes ansiosos de seguirlas.

A pesar de que David afirmó en una entrevista que "trato de entender la posición de otros maestros aún cuando muchas veces no la comparto, por ejemplo cuando dicen que enseñan a pensar o a construir conocimiento cuando los alumnos consultan en un libro, o cuando dicen que los alumnos investigan cuando hacen un laboratorio en una guía que es seguir instrucciones" la realidad del aula presenta algunas discrepancias pues es el maestro quien asigna los problemas a "investigar" que en la mayoría de los casos provienen del libro de texto. "El investigar" se convierte en seguir las instrucciones de la guía de trabajo pues no hay muchas oportunidades de escoger problemas auténticos relevantes para el estudiante. Existe también en este maestro la tendencia a considerar que la verdad se obtiene mediante el seguimiento del método científico pero acorde con Young (1992), no existe un conjunto de conocimientos que aseguren la obtención de "la verdad" por lo tanto esta relación no puede semejarse a la relación proceso-producto.

El documento Ciencias Naturales y Educación Ambiental Marco General intenta proveer orientaciones conceptuales y metodológicas para el diseño y desarrollo curricular El documento resalta la necesidad de entender la enseñanza de la biología de forma diferente.

tenemos que aceptar la necesidad de concebir de una forma diferente la enseñanza de las ciencias: no se trata de transmitir verdades inmutables, sino de darle al estudiante la posibilidad de ver que su perspectiva de mundo no es el mundo, sino una perspectiva de él y una entre las muchas posibles. Enseñar ciencias \{biología\} debe ser darle al estudiante la oportunidad de establecer un diálogo racional entre su propia perspectiva y las demás con el fin de entender de mejor manera el mundo en que vive (Ministerio de Educación Nacional, 1995, p. 14).

\section{CONCLUSIONES}

Por esta razón pensar en la educación en biología de una manera diferente, por ejemplo, de una manera crítica nos trae varias ventajas. Es importante recordar que al criticar la práctica y la teoría de la biología se está criticando también el método, y que la crítica puede ser tanto positiva como negativa y tiende a evidenciar fortalezas y debilidades. Esto nos lleva a pensar en una redefinición de biología, de método, de problema.

Hablar de la educación en biología no significa hablar solamente de las clases y laboratorios de biología en la escuela o de las diferentes metodología empleadas por los 
maestros para enseñar biología Hablar de la educación en ciencias \{biología\} significa también cualquier proceso deliberado que promueva la capacidad de maestros y estudiantes para resolver problemas y que en cierta medida posibilite que los estudiantes sean más grandes que sus maestros (Young, 1992).

Concepciones de biología semejantes y coherentes con ambientes de aprendizaje vivenciados en esta aula están enmarcadas particularmente dentro de una filosofía positivista que constituye un fuerte marco de referencia para jas acciones y expectativas de maestros y estudiantes. Si queremos implementar diferencias en nuestras aulas de clase es importante que el maestro de biología establezca su propio proyecto de investigación para documentar su pensamiento y su práctica educativa y reflexionar críticamente en beneficio del aprendizaje de sus estudiantes.

Del trabajo con este maestro se hace evidente la necesidad de un nuevo paradigma para la enseñanza y el aprendizaje que guíe los cambios en las prácticas de los maestros y de sus estudiantes. Si los maestros son conscientes de las creencias que manejan será factible que la reflexión sobre ellas les motive a generar formas alternativas de pensamiento y acción con los correspondientes cambios curriculares. Por lo tanto la reflexión crítica del maestro en cuanto a su relación con el conocimiento, hecha posible por medio de la investigación acción, mantiene la promesa de avanzar en la educación en biología y de reconocer que existen otras visiones acerca del conocimiento que nos sirven de marco de referencia para hallarle sentido a nuestras acciones docentes.

\section{REFERENCIAS}

ARONOWITZ. S. \& Giroux. H. (1993). Post-modern education. University of Minnesota Press Minneapolis, U.S.A.

CAICEDO. H. (1992). Viabilidad de una línea de investigación sobre enseñanza de las Ciencias. Revista Colombiana de Educación 24. 91-98

CONPES. (1994) Política Nacional de Ciencia y Tecnología Departamento Nacional de Planeación El Salto Social. Documento 2739 Santafé, de Bogotá.

CRONIN-JONES L. (1991). Science teacher beliefs and their influence en curriculum implementation: Two case studies. Journal of Research in Science Teaching, 28(3), 235-250.

GUBA, E. O. \& LINCOLN, Y. S. (1989). Fourth generation evaluation, Newbury Park, CA:SAGE.

MCROBBIE, C. \& TOBIN, K. (1995). Restraints to reform: The congruence of teacher and student actions in a chemistry classroom Journal of Research in Science Teaching, 32(4), 373-385.

Ministerio de Educación Nacional. (1994). Lineamientos Generales de Procesos Curnculares. Hacia la Construcción de Comunidades Educativas Autónomas. Santafé, de Bogotá: Punto EXE. editores. 
REYES-HERRERA L. (1996). An interpretive study of social forces that constrain actions and interactions in a science classroom in Colombia. Doctoral dissertation. The Florida State University, Tallahassee, Florida.

RUTHERFORD, F.J. \& AHLGRENA. (1990). Science for All Americans. New York: Oxford University Press.

SALCEDO, L (1994) Concepciones de los profesores de Química del nivel medio sobre prácticas de laboratorio. En Memorias Congreso Colombiano de Química. Santa Marta.

TOBIN, K. (1993). Constructivist perspectives en teacher learning. In K. Tobin (Ed.). The Practice of Constructivism in Science Education. Washington, D.C.. AAA Press.

TOBIN, K. \& TIPPINS, D. (1993). Constructivism as a referent for teaching and learning. In

Tobin K (Ed) (1993). The Practice of Constructivism in Science Education (pp. 3-21). Washington, D.C.: AAA Press.

TOBIN, K. (1995). Learning from the stories of science teachers. In Haley- Oliphant (Ed.). This year in school science. Washington, D.C.: AAAS Press.

YOUNG, R. (1992). Critical Theory and Classroom Talk. The Language and Education Library 2. Multilingual Matters Ltd. Clevedon, England.

ERICKSON, F. (1986). Qualitative methods in research on teaching. In Merlín C. Wittrock (Ed.) Handbook of Research on teaching: A project of the American Education Research Association (erd ed.) New York: Macmillan, 